\title{
Von Äpfeln und Birnen
}

\section{Christoph Bosshard}

Dr. med., Vizepräsident der FMH, Departementsverantwortlicher Daten, Demographie und Qualität

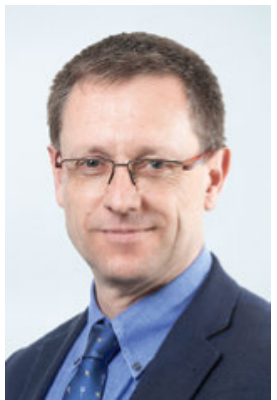

Wie glücklich doch einfache Dinge machen können: «Bring noch ein Kilogramm Brot nach Hause», höre ich, wenn ich mich zum Abendessen anmelde. Wenn ich vor dem Regal stehe, wird die Sache dann oft etwas komplizierter: hell oder dunkel, mit oder ohne Körner? Was einfach tönt, wird plötzlich herausfordernder. Glücklicherweise sind gewisse Entscheidungen in unserem Alltag von überschaubarer Tragweite - andere Dinge sind jedoch anspruchsvoller. Wie entwickelt sich die Demographie der Ärzteschaft? Wie sieht es mit den Arbeitspensen aus? Bilden wir die Summe der ärztlichen Arbeitskraft genügend ab, wenn wir lediglich Köpfe zählen?

Es ist eine gesellschaftliche Tatsache, dass Teilzeitarbeit zunimmt, auch bei Ärztinnen und Ärzten. Sie ist stärker verbreitet bei Medizinerinnen als bei ihren Kollegen. Zwar stellen aktuell die Männer mit 59\% gegen über 41\% Frauen nach wie vor die Mehrheit der berufstätigen Ärzteschaft dar. Dieses Verhältnis wird sich jedoch mittelfristig verändern und langfristig noch zusätzlich akzentuieren: Die Zukunft der Medizin ist klar weiblich, wenn wir unseren Blick auf die Diplomabsolventinnen und -absolventen richten, welche in sechs bis neun Jahren als Fachärztinnen und Fachärzte ins eigenverantwortliche Berufsleben einsteigen.

Der Trend zu mehr Teilzeitarbeit wird anhalten, da die Medizin der Zukunft klar weiblich ist.

Eine weitere Herausforderung besteht darin, dass die Definition eines Vollzeitpensums nicht einheitlich geklärt ist. Ich erinnere mich noch gut an die folgende Aussage: «Der Tag hat 24 Stunden, dann gibt es noch die Nacht, und wenn's dann immer noch nicht reicht, machen wir etwas Überzeit.» Die Erhebung in myFMH erfasst die gearbeiteten Halbtage und versucht, die Dauer eines halben Arbeitstags mit vier bis sechs Stunden zu erfassen. Ob dies der Heterogenität der Fachrichtungen, aber auch der Altersklassen der Ärzteschaft genügend gerecht wird, gilt es in Zukunft noch näher zu untersuchen, wenn wir nicht Äpfel mit Birnen vergleichen wollen. Denn dies geschieht leider allzu oft, wie ein kürzlich beobachtetes Beispiel aus dem Kanton Bern illustriert: Ein Kinderarzt hat nach reich befrachtetem Berufsleben das Glück, eine Nachfolgelösung für seine Praxis zu finden - fünf Ärztinnen in Teilzeit führen die Arbeit weiter. Die KopfStatistik spricht dann von einer Verfünffachung der Versorgung.

Hier kommen wir beim nächsten Stichwort an, der Versorgung. Diese beinhaltet nämlich nicht nur die Seite der Leistungserbringer, sondern auch diejenige der Leistungsbezüger. Wenn wir uns der demographischen Entwicklung unserer Bevölkerung zuwenden, dann sehen wir, dass die Altersklasse im Bereich 75+ in Zukunft gegenüber der übrigen Bevölkerungsentwick-

\section{Statt Köpfe zu zählen, sind Vollzeitäquivalente} adäquate Orientierungshilfen für die Planung der medizinischen Versorgung.

lung überproportional zunehmen wird. Diese Entwicklung widerspiegelt sich aktuell in der Diskussion um die Altersvorsorge. Dass der Solidargedanke bereits hier im Rahmen der auch gesunden Gesellschaft auf dem Prüfstand steht, erachte ich als unbestreitbar. Zusätzlich wird die Situation mit dem Umstand belastet, dass der Anteil der Kosten im Gesundheitswesen über die Altersklassen hinweg in der erwähnten Klasse 75+ überproportional gewichtet ist. Wir stehen also vor zwei sich multiplizierenden überproportionalen Faktoren auf der Leistungsbezüger-Seite, welche auch bei unverändertem Pro-Kopf-Bedarf in der Gesamtbevölkerung die Versorgungslage in Zukunft beeinflussen. Hier ist die Politik und Verwaltung gefragt, Perspektiven aufzuzeigen. Auf Ebene der Ärzteschaft nimmt die FMH diese Verantwortung wahr und trägt ihren Teil dazu bei, unser Gesundheitswesen auf Kurs zu halten. Ich danke jedem Mitglied ganz herzlich, welches auch mit der Aktualisierung seiner Daten in myFMH dazu beiträgt. 